\title{
Surface Functionalization of SU-8 Vertical Waveguide for Biomedical Sensing: Bacteria Diagnosis ${ }^{\dagger}$
}

\author{
Yu Xin 1, Grégory Pandraud 2, Linda Otten ${ }^{3}$, Yongmeng Zhang ${ }^{4}$ and Paddy French 5,* \\ 1 State Key Laboratory of NBC Protection for Civilian, Beijing 102205, China; xinyu_0619@163.com \\ 2 Else Kooi Laboratory, TU Delft, 2628CT Delft, The Netherlands; G.Pandraud@tudelft.nl \\ 3 Department of Biotechnology, TNW, TU Delft, 2629HZ Delft, The Netherlands; L.G.Otten@tudelft.nl \\ College of Mechatronics Engineering and Automation, NUDT, Changsha 410073, China; zymnudt@163.com \\ 5 Electronic Instrumentation Lab, EWI, TU Delft, 2628CD Delft, The Netherlands \\ * Correspondence: P.J.French@tudelft.nl; Tel.: +31-15-2784729 \\ + Presented at the Eurosensors 2018 Conference, Graz, Austria, 9-12 September 2018.
}

Published: 16 November 2018

\begin{abstract}
In this paper, we present an SU-8 based evanescent waveguide with a vertical structure as a biomedical sensor. The waveguide is designed vertically to generate evanescent waves on both left and right surfaces for sensing. It is fabricated by E-beam lithography with only one-step process which has the advantage of a better surface quality compared with commonly used dry etching methods. Furthermore, fabrication time and cost is cut down greatly. The surface of the designed waveguide can be functionalized with antibodies to immobilize specific bacteria on it. After surface functionalization and incubation with E. coli solutions of different concentrations, the waveguides absorption was measured. The results demonstrate that the waveguide is sensitive to E. coli concentration changes. In addition, tapers were designed and added to the waveguide to relieve the alignment tolerance for the aim of making a plug-and-play bedside diagnostic system.
\end{abstract}

Keywords: SU-8; vertical waveguide; biomedical sensing; surface functionalization

\section{Introduction}

With the increasing bacteria hazards in environment, food system, and health care, biomedical sensors have raised a lot of attention since its vast application in chemical and biomedical sensing. An ideal biosensor should be able to provide fast, accurate, sensitive and cost-effective analysis [1], with the potential of easily operation and integration ability at the same time. Waveguide based biomedical sensor stands out for its merits of high sensitivity, integration ability, free from electromagnetic influence, material selection flexibility, etc. Furthermore, optical systems have the merit of fast response which is a crucial factor in bedside diagnosis. The waveguide sensor in this paper is proposed for monitoring bacteria in fluid, e.g., the existence and concentration of E. coli in patients' drain fluid after colon anastomosis. The waveguide utilizes evanescent wave for sensing, which is sensitive to the targets on the surface of the waveguide. A vertical waveguide structure is used in this design to increase the waveguide sensitivity. The surface of the waveguide can then be functionalized with antibodies to fix targets on it for detection.

\section{Material and Design}

The waveguide was designed vertically to generate evanescent waves on both left and right surfaces for sensing, as shown in Figure 1a. This increases the sensing area and optical energy distributed in the cladding to interact with immobilized targets. Both aspects will increase waveguide sensitivity. The fundamental mode is simulated and shown in Figure 1b. SU-8 is chosen 
as waveguide core material because of its suitable optical properties: transparent above $400 \mathrm{~nm}$ and with a refractive index of 1.57 at $1.3 \mathrm{um}$ wavelength. It is a photoresist that can be fabricated by E-beam lithography, a maskless direct writing method which can guarantee fast prototyping, sub-micron and high precision fabrication [2]. Compared with traditional materials in the fabrication of this proposed vertical large height-width ratio structure, SU-8 outstands other materials as no dry etching is needed, which guarantees the side wall surface quality. Moreover, it is very stable after polymerization [3], which is crucial as a chemical or biomedical sensor material. After chemical treatment, the surface of SU-8 can be functionalized with antibodies [4-9] and this enables its applications in biomedical field.

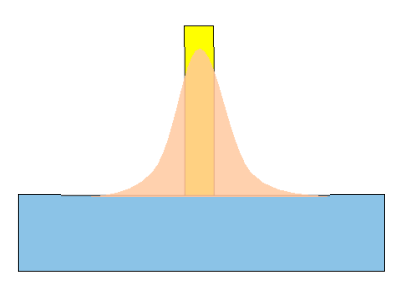

(a)

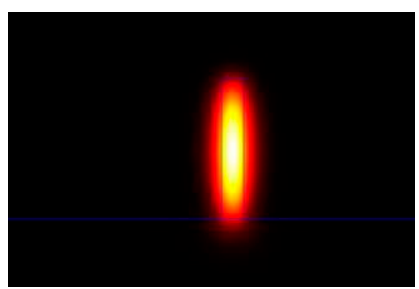

(b)

Figure 1. (a) The cross section of designed SU-8 waveguide with evanescent waves on both surfaces;

(b) Simulated fundamental mode in the waveguide.

SU-8 waveguide structure parameters were designed by COMSOL simulation in previous research [10], optimizing optical energy for sensing and at the same time considering fabrication feasibility. When fabricating waveguides, $\mathrm{SiO}_{2}$ was first thermally grown on $\mathrm{Si}$ wafer as an isolation layer. Afterwards, the waveguides were fabricated by E-beam lithography in Kavli by Raith EBPG-5200. Then, a slow ramp-up and cool-down baking process were used to improve the adhesion of SU-8 to the substrate. After development, a protection photoresist layer was coated on the top and then the wafer was diced into chips ready for the next step.

\section{Surface Functionalization}

Surface functionalization is the process of attaching antigen/antibody to the surface of a waveguide. This includes surface modification and antibody/antigen immobilization [11]. Surface modification is aimed at creating a surface with the property that can react with the antibodies afterwards. The general procedure starts with creating -OH groups on the surface and then creating amine groups on the previous layer. Afterwards, by applying glutaraldehyde on the chip, the amine groups and antibodies can be bridged. After the antibodies are immobilized on the surface, the wafer is successfully functionalized and is ready for antigen immobilization.

A functionalization method was designed and demonstrated to be effective to fix antibodies and capture E. coli on the SU-8 surface. The procedure is illustrated in Figure 2.

\section{Step 1: Plasma treatment}

The SU-8 surface is activated by oxygen plasma for $5 \mathrm{~min}$ at a pressure of $200 \mathrm{mTorr}$ and a power of $30 \mathrm{~W}[4,12]$. Through plasma treatment, the epoxy group is opened and -OH groups are created on the SU-8 surface.

Step 2: APTES Treatment

This step creates amine groups on the surface. APTES is diluted in $75 \%$ ethanol and $25 \%$ water at a 1:100 ratio. Then the chips are incubated overnight at room temperature.

Step 3: Glutaraldehyde treatment

The wafer is activated using a $1.2 \%(v / v)$ glutaraldehyde solution in $1 \times$ phosphate buffer saline (1× PBS) at room temperature for $2 \mathrm{~h}$. Then it is washed excessively with DI water and $1 \times$ PBS. Glutaraldehyde is a di-aldehyde, which contains 2 active aldehyde moieties (=CHO). These aldehydes react with amine groups which are present on the APTES and on the antibody. In this way, glutaraldehyde bridges the APTES with the antibody.

Step 4: Antibody immobilization 
The original antibody solution is diluted in $1 \times$ PBS to make an antibody solution of $10 \mu \mathrm{g} / \mathrm{mL}$. The solution on the surface is dropped and incubated for $1 \mathrm{~h}$ at room temperature.

Step 5: Antigen reaction

This step is to create an E. coli-antibody association, through the process of applying E. coli on the functionalized specimens and incubating the bacteria for a defined time period $(2 \mathrm{~h})$. Then, wash the incubated specimens with DI water.

After the steps designated above, the chips are functionalized with E. coli and ready for the following optical measurements.

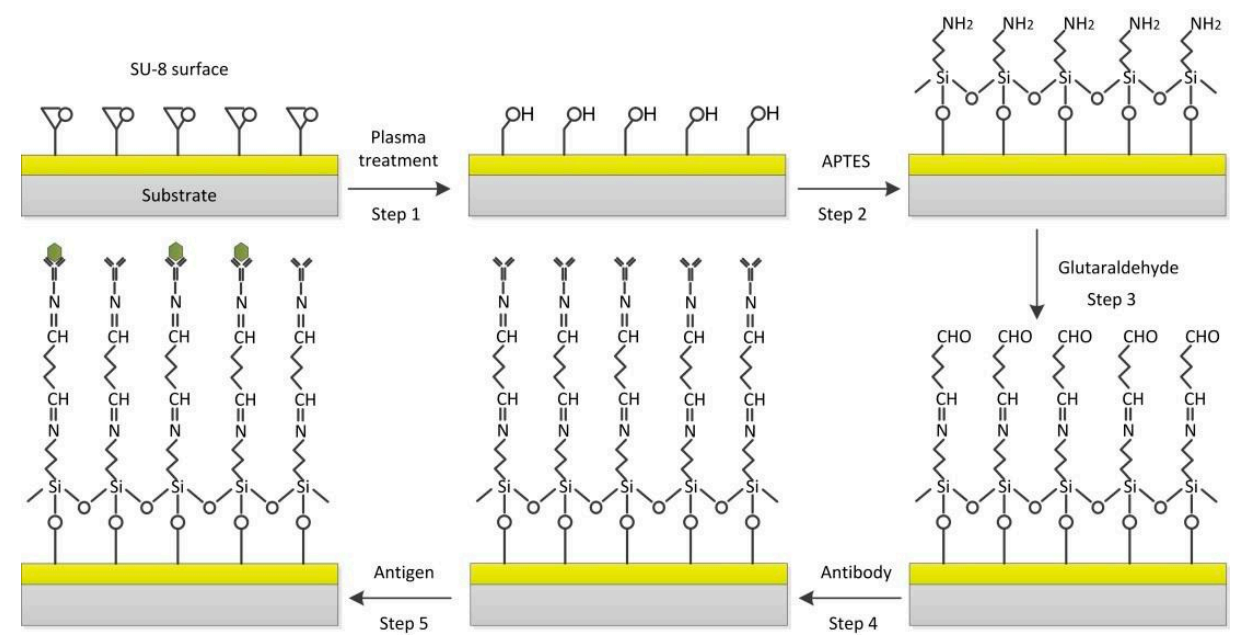

Figure 2. SU-8 surface functionalization process.

\section{Measurements}

After functionalization, the treated SU-8 waveguide chips were incubated with E. coli solutions with concentrations of $10^{2} \mathrm{CFU} / \mathrm{mL}, 10^{3} \mathrm{CFU} / \mathrm{mL}, 10^{4} \mathrm{CFU} / \mathrm{mL}, 10^{5} \mathrm{CFU} / \mathrm{mL}, 10^{6} \mathrm{CFU} / \mathrm{mL}$ for $2 \mathrm{~h}$ to immobilize E. coli on the waveguide sensing surface. A negative control group was also set up with the same chemical treatment but without antibody treatment. The incubation is shown in Figure 3. When incubated with $E$. coli solutions of different concentrations, different numbers of $E$. coli will be fixed on the surface. After flush away the excess uncaptured E. coli, the waveguide with captured bacteria on the surface will absorb more optical energy than the reference waveguide without antibody treatment. In this way, a bacteria concentration-absorption relationship can be obtained. After calibration, the waveguide can be used to detect unknown concentration solutions.

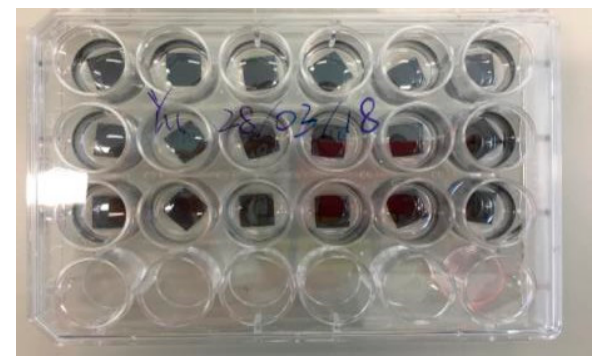

Figure 3. Incubating SU-8 waveguides with E. coli solutions of different concentrations.

\section{Results}

After incubation, the chips were fixed and then measured. The absorption of waveguides with different E. coli treatments was measured. The results are shown in Figure 4. This figure shows an increasing trend with the increase in E. coli concentration, which demonstrates its sensitivity to $E$. coli. The waveguide is able to detect bacteria when the concentration is $10^{2} \mathrm{CFU} / \mathrm{mL}$. Moreover, it becomes more significant when the concentration reaches $10^{4} \mathrm{CFU} / \mathrm{mL}$. The sign of leakage in 
patients is normally when the E. coli concentration quickly ramps up to $10^{5} \mathrm{CFU} / \mathrm{mL}$ in the drain fluid after surgery [13]. The proposed system meets therefore the medical requirements and further improvements will make such a system vital for early diagnostics.

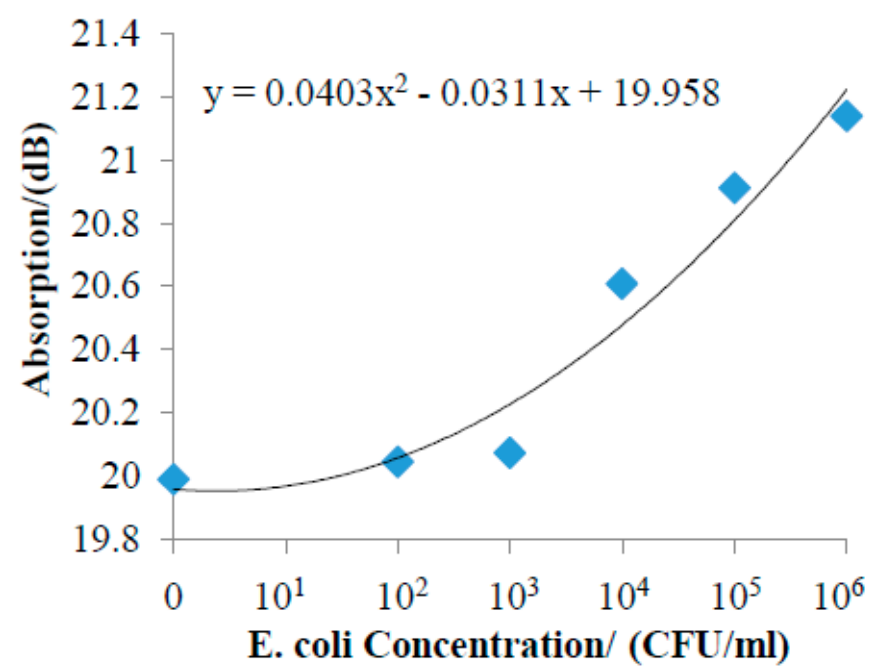

Figure 4. Sensitivity measurement of the SU-8 waveguide with different $E$. coli concentrations.

Author Contributions: Y.X. conceived and designed the experiments, performed the experiments; G.P. and P.F. discussed and gave suggestions on the design and experiments; Y.X. and Y.Z. conducted the simulations; Y.X. and L.O. did the biomedical treatments; Y.X. wrote the paper and P.F. and G.P. revised the paper.

Acknowledgments: The authors would like to acknowledge to staff from EKL, Kavli Lab and chemical department for their assistance during the fabrication and the discussion during that time. The first author $\mathrm{Yu}$ Xin would like to acknowledge the Chinese Scholarship Council (CSC) and the Micro-nano System Lab in National University of Defense Technology to support her doing research in TU Delft.

Conflicts of Interest: The authors declare no conflict of interest.

\section{References}

1. Estevez, M.C.; Alvarez, M.; Lechuga, L.M. Integrated optical devices for lab-on-a-chip biosensing applications. Laser Photonics Rev. 2012, 6, 463-487.

2. Sum, T.C.; Bettiol, A.A.; van Kan, J.A.; Watt, F.; Pun, E.Y.B.; Tung, K.K. Proton beam writing of low-loss polymer optical waveguides. Appl. Phys. Lett. 2003, 83, 1707-1709.

3. Microchem. SU-8 3000 Permanent Epoxy Negative Photoresist. Available online: http://www. microchem.com/pdf/SU-8 3000 DataSheet.pdf (accessed on 5 October 2017).

4. Grimaldi, I.A.; Testa, G.; Persichetti, G.; Loffredo, F.; Villani, F.; Bernini, R. Plasma functionalization procedure for antibody immobilization for SU-8 based sensor. Biosens. Bioelectron. 2016, 86, 827-833.

5. Deepu, A.; Sai, V.V.R.; Mukherji, S. Simple surface modification techniques for immobilization of biomolecules on SU-8. J. Mater. Sci. Mater. Med. 2009, 20 (Suppl. 1), 25-28.

6. Shew, B.Y.; Cheng, Y.C.; Tsai, Y.H. Monolithic SU-8 micro-interferometer for biochemical detections. Sens. Actuators A Phys. 2008, 141, 299-306.

7. Tao, S.L.; Popat, K.C.; Norman, J.J.; Desai, T.A. Surface modification of SU-8 for enhanced biofunctionality and nonfouling properties. Langmuir 2008, 24, 2631-2636.

8. Jiang, L.; Gerhardt, K.P.; Myer, B.; Zohar, Y.; Pau, S. Evanescent-Wave Spectroscopy Using an SU-8 Waveguide for Rapid Quantitative Detection of Biomolecules. J. Microelectromech. Syst. 2008, 17, 14951500 .

9. Joshi, M.; Pinto, R.; Rao, V.R.; Mukherji, S. Silanization and antibody immobilization on SU-8. Appl. Surf. Sci. 2007, 253, 3127-3132.

10. Xin, Y.; Pandraud, G.; van Langen-Suurling, A.; French, P. Tapered SU8 waveguide for evanescent sensing by single-step fabrication. In Proceedings of the 2017 IEEE SENSORS, Glasgow, UK, 29 October-1 November 2017; pp.1-3. 
11. Boiragi, I.; Makkar, R.; Choudhury, B.D.; Mallik, A.; Chalapathi, K.; Sebastian, J. SU-8 Polymer Based Waveguide Biochemical Sensor for Medical Diagnostic Application. In Proceedings of the ICOP 2009-International Conference on Optics and Photonics CSIO, Chandigarh, India, 30 October-1 November 2009; pp. 2-5.

12. Hamdi, F.S.; Woytasik, M.; Couty, M.; Francais, O.; le Pioufle, B.; Dufour-Gergam, E. Low temperature irreversible poly(DiMethyl) Siloxane packaging of silanized SU8 microchannels: Characterization and lab-on-chip application. J. Microelectromech. Syst. 2014, 23, 1015-1024.

13. Komen, N.; Slieker, J.; Willemsen, P.; Mannaerts, G.; Pattyn, P.; Karsten, T.; de Wilt, H.; van der Harst, E.; van Leeuwen, W.; Decaestecker, C.; et al. Polymerase chain reaction for Enterococcus faecalis in drain fluid: The first screening test for symptomatic colorectal anastomotic leakage. The Appeal-study: Analysis of Parameters Predictive for Evident Anastomotic Leakage. Int. J. Colorectal Dis. 2013, 29, 15-21.

(C) 2018 by the authors. Licensee MDPI, Basel, Switzerland. This article is an open access article distributed under the terms and conditions of the Creative Commons Attribution (CC BY) license (http://creativecommons.org/licenses/by/4.0/). 